\title{
IMPACTO Y BENEFICIOS DERIVADOS DEL USO DE CRM POR PARTE DE LAS EMPRESAS DEL SECTOR DE LA CONSTRUCCIÓN EN ESPAÑA (PROYECTO CRM-CONSTR-ES)
}

IMPACT AND BENEFITS DERIVED FROM THE USE OF CRM BY
COMPANIES IN THE CONSTRUCTION SECTOR IN SPAIN (CRM-
CONSTR-ES PROJECT)

\author{
Vicente Guerola-Navarro \\ Departamento de Organización de Empresas. \\ Universitat Politècnica de València. Valencia, (España). \\ E-mail: viguena@upv.es ORCID: https://orcid.org/0000-0002-0367-6319 \\ Raul Oltra-Badenes \\ Departamento de Organización de Empresas. \\ Universitat Politècnica de València. Valencia, (España). \\ E-mail: rauloltra@doe.upv.es ORCID: https://orcid.org/0000-0002-1522-8230 \\ Hermenegildo Gil-Gomez \\ Departamento de Organización de Empresas. \\ Universitat Politècnica de València. Valencia, (España). \\ E-mail: hgil@ai2.upv.es ORCID: https://orcid.org/0000-0002-7985-2454
}

Recepción: 02/09/2021 Aceptación: 22/10/2021 Publicación: 24/11/2021

\section{Citación sugerida:}

Guerola-Navarro, V., Oltra-Badenes, R., y Gil-Gomez, H. (2021). Impacto y beneficios derivados del uso de CRM por parte de las empresas del sector de la construcción en España (Proyecto CRM-CONSTR-ES). 3 C Empresa. Investigación y pensamiento crítico, 10(4), 17-41. https://doi.org/10.17993/3cemp.2021.100448.17-41 


\section{RESUMEN}

Se presenta en este artículo la estructura y plan de acción de un proyecto (CRM-CONSTR-ES) cuyo objetivo es analizar, dentro del sector de la construcción en España, cual es el impacto que tiene usar soluciones Customer Relationship Management (CRM) para la gestión de las relaciones con los clientes. Se pretende comprobar empíricamente si es real la presunción de un escaso ratio de adopción de soluciones CRM por parte de estas empresas, y aportar criterios que demuestren la valía de estas soluciones como elementos de gestión claves para mejorar la competitividad de estas empresas. El estudio no abarca solamente la declaración de intenciones del proyecto, sino también el grado de avance del mismo y los resultados iniciales. Con la finalidad de hacer lo más práctico y aplicable posible este estudio, se ha trabajado juntamente con un Partner tecnológico de referencia, especializado no solo en el despliegue de soluciones CRM sino también en el sector de la Construcción. Tras elegir la muestra de empresas representativas del sector, se ha diseñado un formulario para la recogida de datos, que serán analizados siguiendo el método científico. En una etapa inicial del proyecto, el ratio de respuesta y las primeras conclusiones confirman un bajo nivel de adopción de CRM por parte de estas empresas, así como un elevado grado de satisfacción por parte de las que usan CRM como herramienta de gestión de sus relaciones con los clientes.

\section{PALABRAS CLAVE}

Construcción, Transformación Digital, Customer Relationship Management, CRM, Competitividad, Desempeño Organizacional. 


\section{ABSTRACT}

This article presents the structure and action plan of a project (CRM-CONSTR-ES) whose objective is to analyze, within the construction sector in Spain, what is the impact of using Customer Relationship Management (CRM) solutions for managing customer relationships. The aim is to empirically test whether the presumption of a low rate of adoption of CRM solutions by these companies is real, and to provide criteria that demonstrate the value of these solutions as key management elements to improve the competitiveness of these companies. The study does not only cover the declaration of intentions of the project, but also the degree of progress of the project and the initial results. In order to make this study as practical and applicable as possible, we have worked together with a leading technological Partner, specialized not only in the deployment of CRM solutions but also in the Construction sector. After choosing the sample of companies representing the sector, a form has been designed to collect data, which will be analyzed following the scientific method. In an initial stage of the project, the response rate and the first conclusions confirm a low level of adoption of CRM by these companies, as well as a high degree of satisfaction on the part of those who use CRM as a tool for managing their relationships with the clients.

\section{KEYWORDS}

Construction, Digital Transformation, Customer Relationship Management, CRM, Competitiveness, Firm Performance. 


\section{INTRODUCCIÓN}

El uso de las TIC (Tecnologías de la Información y Comunicación), por parte de las empresas, como factor determinante para potenciar sus capacidades de innovación y adaptación al entorno (Nambisan $e t$ al., 2017; Ribeiro-Navarrete, 2021), es uno de los factores clave en el proceso de transformación digital que están afrontando las empresas de todo el mundo en su afán por sobrevivir y mantener su posición en el mercado (Dew et al., 2011; AlQershi et al., 2020).

La globalización, que facilita el acceso de todos los actores del mercado, no solo a información (en cantidad y en calidad) sino también a las propias transacciones mercantiles, hace que toda empresa que quiera perdurar en el mercado y mantener su ventaja competitiva, deba afrontar cuanto antes su propio proceso de transformación digital (Kane et al., 2015).

En este sentido, y no sólo dentro del mercado español, la experiencia y los estudios muestran que existen ciertas barreras y dificultades, relacionadas con la inercia y la resistencia al cambio, que actúan como barreras de entrada de ciertos sectores a este tipo de proceso de transformación (Müller et al., 2018) que va a ser clave para su subsistencia en un mundo cada vez más dinámico y exigente para con sus actores, en especial dentro de las Pequeñas y Medianas Empresas (PYMEs).

Uno de los sistemas de gestión que se presenta como más eficiente y de mayor impacto para que las empresas puedan afrontar con éxito sus procesos de transformación digital, es la solución tecnológica CRM, que surge (junto con los sistemas ERP) como una de las soluciones de negocio con mayor impacto y relevancia en la actualidad (Vicedo et al., 2020; Gil-Gomez et al., 2020). CRM es una de las tecnologías clave en este proceso de transformación digital, tanto por las mejoras de gestión internas que puede y debe atraer a la empresa (en cuanto a mejora y más eficiente gestión de las relaciones con los clientes) como por el impacto que ello puede tener en el mantenimiento y potenciamiento de la ventaja competitiva de la empresa en el mercado (Guerola-Navarro et al., 2020b). 
En las últimas décadas, CRM ha sido una de las soluciones tecnológicas más abordadas desde el punto de vista de la investigación científica (Guerola-Navarro et al., 2020a; Guerola-Navarro et al., 2021a), no sólo pro su amplio y exponencial crecimiento en el número de despliegues en las empresas, sino también por las modernas teorías del marketing relacional que ponen a la gestión de las relaciones con los clientes en el centro de las decisiones de toda empresa que quiera sobrevivir en el mercado (Araújo et al., 2018).

No obstante, y pese a que se han realizado estudios recientes sobre el impacto de uso de CRM en sectores con escaso nivel de adopción de CRM en diferentes entornos geográficos (Guerola-Navarro et al., 2020c; Guerola-Navarro et al., 2021b; Iazzi et al., 2013; Belias et al. 2018; Crescimanno et al., 2017; Ferrer-Lorenzo et al., 2017), hay muchos sectores en los que queda mucho camino por recorrer en el estudio sobre el impacto de uso de CRM sobre sus resultados. En la presente ocasión, se presenta un proyecto de investigación que comprende un estudio empírico sobre el impacto del uso de CRM sobre el firm performance en el sector de la Construcción española.

Con la finalidad de realizar dicho estudio empírico de impacto de uso de CRM e innovación sobre firm performance, este proyecto se inicia con la definición del marco teórico sobre el que se desarrolla el estudio, tanto desde el punto de vista del sector de la Construcción en España (objeto de estudio) como desde el punto de vista de la tecnología CRM (tanto los sistemas tecnológicos CRM, como las principales dimensiones que lo identifican, inclusive la cultura de gestión de relaciones con los clientes). La siguiente etapa es la descripción taxonómica de la base de datos sobre la que se realiza el estudio, así como el proceso de selección de la muestra representativa de la población. En la fase siguiente se explica cuál es la metodología utilizada para la validación empírica de la relación entre las principales variables que definen el modelo de investigación elegido, para finalmente presentar los resultados, su discusión e interpretación para obtener las conclusiones empíricas de valor académico y empresarial. 


\section{MARCO CONCEPTUAL}

\subsection{CUSTOMER RELATIONSHIP MANAGEMENT (CRM)}

CRM ha demostrado ser una de las soluciones de gestión empresarial de mayor crecimiento en las últimas décadas, tanto en número de despliegues como en materia de interés para los investigadores (Guerola-Navarro et al., 2020a; Gutiérrez Guevara y Romero Vargas, 2021; Guerola -Navarro et al., 2021). El reconocimiento de los beneficios esperados del uso de CRM en el marco de la fidelización del cliente y el mantenimiento de los beneficios a largo plazo esperados mediante el refuerzo de la ventaja competitiva en el mercado (Gil-Gomez et al., 2020) ha dotado a CRM con el halo de una herramienta de gestión clave para la supervivencia de las empresas en el mercado (Rahmadi et al., 2021). Wahlberg et al. (2009) proporciona una imagen clara del CRM como un foco creciente de investigación científica, con una batería de estudios de referencia todavía no muy abundante, pero con una base de datos bibliográfica en continuo crecimiento que hace del CRM uno de los pilares básicos de estudio en el campo de la búsqueda de la excelencia empresarial a través de estrategias de gestión centradas en el cliente.

A pesar de que CRM nació como una solución básica para la automatización de la fuerza de ventas (Buttle, 2004), su evolución hacia modelos de gestión compleja de las relaciones con los clientes ha hecho del CRM un modelo de gestión que aporta tanto componentes de cultura empresarial como de tecnología. gestión, todos ellos directamente relacionados con la mejora del desempeño de la empresa y con ello de los resultados del negocio (Guerola-Navarro et al., 2020b). Chen \& Popovich, (2003) ya propusieron un modelo basado en los tres módulos que componen cualquier solución CRM: Ventas, Marketing y Servicios (Atención al Cliente y Soporte). Las teorías de gestión modernas basadas en estrategias centradas en el cliente han demostrado ser las más eficientes en los esfuerzos de las empresas por adaptar sus cadenas de valor a las necesidades y expectativas de los clientes, en línea con el propósito estratégico del CRM como herramienta de gestión. relaciones con los clientes (Joo, 2007). Huang y Lin (2005) proponen CRM como una de las herramientas clave para lograr la lealtad del cliente y que su 
confianza en la asociación a largo plazo trae beneficios operativos tanto para la empresa como para los clientes.

Como muestran las teorías modernas sobre el impacto que el marketing relacional tiene en la lealtad del cliente (Kurniawan et al., 2021), se ha demostrado que la lealtad del cliente tiene un impacto esperado positivo y decisivo en el desempeño de la empresa. (Ramanathan et al., 2017), por lo que se puede esperar que el despliegue de soluciones CRM en empresas de diferentes sectores productivos pueda desencadenar mejoras en el desempeño firme de las empresas que lo utilizan (Izquierdo et al., 2015; Guerola-Navarro et al., 2020c).

\subsection{CRM Y EL SECTOR DE LA CONSTRUCCIÓN}

Si bien es cierto que el proceso de transformación digital está afectando a todos los sectores, la construcción ha sido tradicionalmente un sector reactivo en la adopción y uso de la tecnología digital. A pesar de ser un sector altamente tecnológico, los datos indican que no se ha percibido el valor de la tecnología en un entorno digital, en la misma medida que en otros sectores, como el uso de software de gestión para el control de proyectos y costos (Marquesme, 2021). Siempre ha tenido una tendencia hacia la tecnología que podríamos calificar como "tecnología mecánica" (maquinaria, automóviles, herramientas, etc.).

Marquesme (2021) concluye que el sector de la construcción es uno de los menos digitalizados de España en la actualidad, situándose solo por delante del sector terciario, la pesca y la agricultura. La mayoría de los procesos en este sector tienen un componente mecánico manual, es un sector donde la mano de obra sigue siendo muy importante. Esta situación supone para las empresas, no solo en este sino en todos los sectores, un enorme sobrecoste en gestión y desvíos por falta de procesos automatizados que aporten valor a la gestión diaria. La tecnología suele verse como un coste o gasto para la empresa, especialmente toda aquella tecnología vinculada a un entorno digital. Cualquier iniciativa para demostrar que la tecnología digital es una inversión rentable es, por tanto, de gran importancia. 


\subsection{ESTUDIOS PREVIOS SOBRE CRM Y EL SECTOR DE LA CONSTRUCCIÓN}

El tema del impacto del uso de CRM en los resultados dentro del sector de la construcción no ha sido muy estudiado y hay relativamente pocas publicaciones al respecto. A pesar de que CRM ha sido ampliamente aceptado y aplicado con éxito en una variedad de sectores, ha habido muy pocos esfuerzos de investigación en el campo de CRM en la industria de la construcción. Sin embargo, existen ciertos estudios que tienen aspectos muy relevantes para contextualizar el presente estudio.

Respecto al sector de la construcción en general, Sear et al. (2007) presenta un caso de estudio en el que se evalúan los cinco factores de éxito que son claves para el éxito en las implementaciones de CRM en la Industria de la Construcción: implementar una estrategia comercial centrada en el cliente; crear una estructura organizativa compatible con CRM; establecer una cultura organizacional experta en CRM; asegurar el compromiso de la alta dirección; y definir medidas de éxito de CRM (Imhoff, 2001). Preece et al. (2015) revisa los beneficios y desafios en las implementaciones de CRM en organizaciones de construcción, y proporciona una revisión de la filosofía y tecnología de CRM considerando las implicaciones, beneficios y desafios para las organizaciones de construcción a un nivel estratégico comercial y operativo. En un mercado dinámico, en constante cambio y altamente competitivo, la implementación de GRM a lo largo del ciclo de vida de los activos puede proporcionar una gestión más eficaz de los clientes actuales y potenciales. Todo ello, dentro de la dinámica del sector de la construcción, debe orientar los esfuerzos del sector hacia un trabajo más colaborativo entre empresa y clientes.

En el área de la ingeniería civil, Melovic et al. (2015) busca las condiciones para incrementar la competitividad de las empresas, apareciendo el CRM como clave para cambiar el enfoque del producto al comprador y de esa manera construir relaciones de calidad, de largo plazo y rentables entre empresas y clientes, como más forma eficiente de mantener la ventaja competitiva en el mercado. La implementación de los sistemas GRM en este tipo de empresas está en correlación positiva directa con los resultados del negocio, enfocando todos los esfuerzos en el comprador y desplegando estrategias adecuadas de creación de relaciones a largo plazo, sostenibles y rentables con los compradores. De la misma forma, 
para la industria de la ingeniería civil, y enfocado principalmente al alcance básico de automatización de la fuerza de ventas, Jocovic et al. (2014) plantea que el CRM debe servir para planificar y gestionar procesos de ventas y fortalecer la competitividad al registrar todas las interacciones de la empresa con sus compradores y proveedores. Junto con las mismas tendencias, Okrepilov y Melovic (2015) también se esfuerzan por cambiar el enfoque en el sector de la ingeniería civil del producto al cliente, para crear relaciones sostenibles y beneficiosas para toda la vida con los clientes.

No existen estudios en la literatura que se refieran al impacto del uso de CRM en el desempeño de la empresa en empresas dedicadas a la construcción, ni, en consecuencia, vincular el CRM con la Innovación y el Desempeño de la Firma, lo que da originalidad y justifica el interés investigador de este estudio.

\section{EL PROYECTO CRM-CONSTR-E}

\subsection{DEFINICIÓN DE OBJETIVOS Y PLAN DE TRABAJO}

\section{$\underline{\text { Objetivos }}$}

El objetivo principal, y que engloba a todos los demás, es analizar cual es el grado de adopción de las soluciones tecnológicas CRM por parte de las empresas del sector de la construcción en España, además de los beneficios de gestión que este tipo de soluciones están aportando a las empresas que lo usan, con el objetivo final de aportar argumentos científicamente obtenidos sobre los beneficios esperados del despliegue y uso de dichas soluciones en las empresas de dicho sector.

Comprobar si efectivamente, y tal y como se presupone y se asume, este sector es uno de los que menor grado de digitalización han afrontado (Marquesme, 2021), es el primer paso para tratar de analizar y demostrar si el uso de soluciones CRM puede ayudarles en su proceso de transformación digital, modernización, y mejora de la gestión empresarial. 
Los objetivos concretos, tal y como aparecen en la Figura 1, que se plantéan en este ámbito de trabajo de investigación sobre el sector de la construcción en España son:

1. Con la ayuda de bases de datos oficiales y de Partners tecnológicos de referencia y de elevada solvencia en el mercado, identificar la muestra de estudio apropiada para validar el impacto de uso de CRM sobre el desempeño organizacional de las empresas del sector que lo usan internamente para gestionar sus relaciones con sus clientes.

2. Seleccionar el modelo de investigación adecuado para validar las condiciones y resultados de impacto del uso de CRM sobre los resultados empresariales.

3. Realizar el estudio empírico que demuestre en qué condiciones el uso de CRM trae a la empresa mejoras en el desempeño organizacional.

Figura 1. Objetivos del estudio.

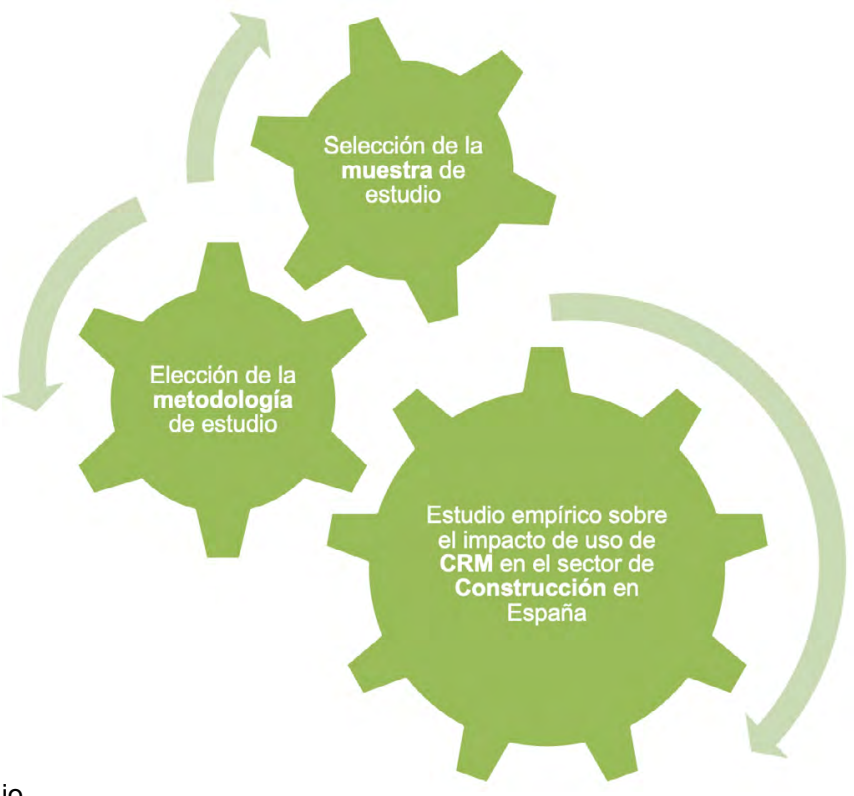

Fuente: elaboración propia. 


\section{Plan de trabajo}

El presente proyecto se estructura siguiendo las fases que se muestran en la Figura 2.

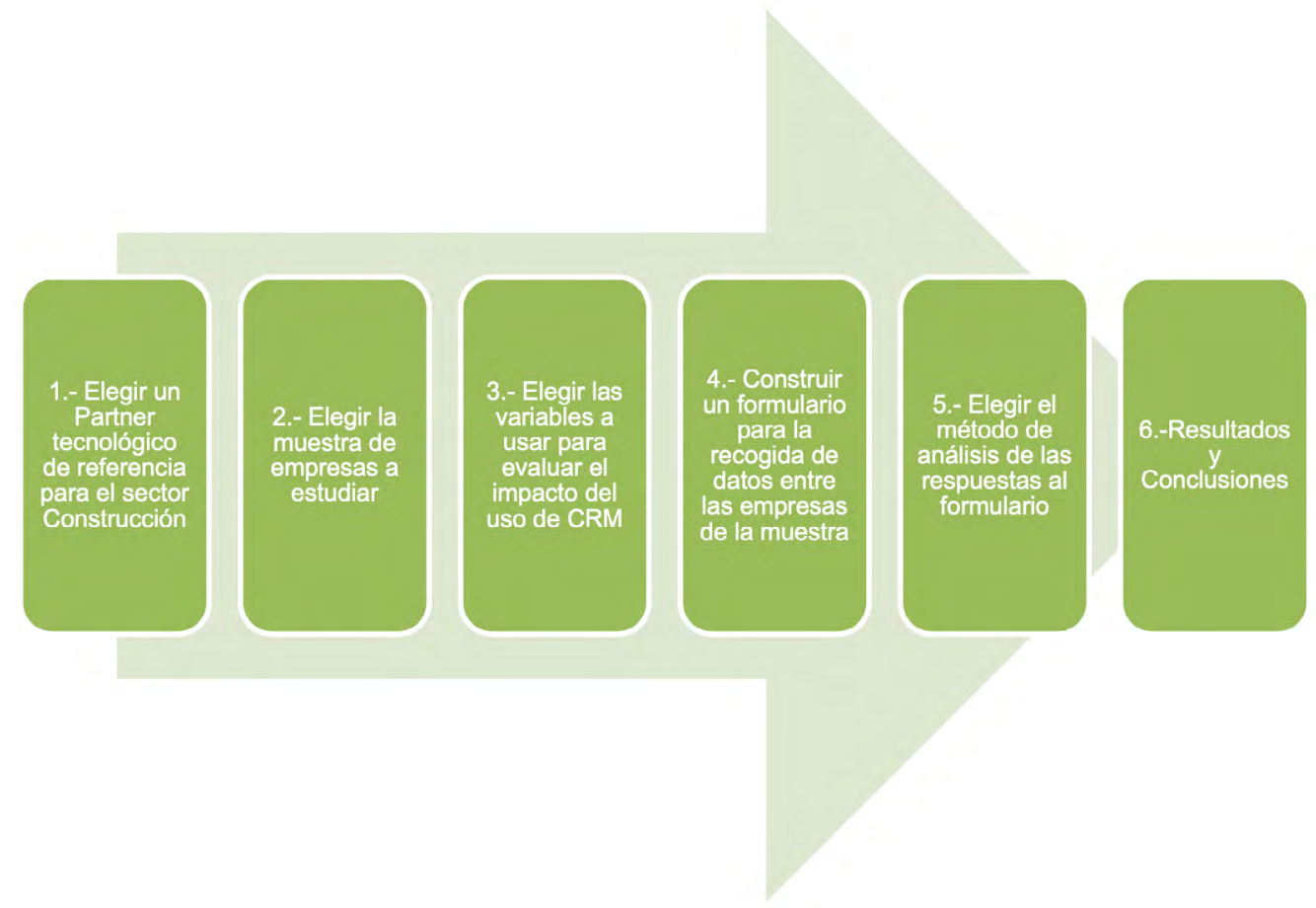

Figura 2. Plan de trabajo.

Fuente: elaboración propia.

Del espíritu claramente práctico y aplicado del presente proyecto, cuya finalidad última es aportar criterios de valor a las empresas del sector que afronten la decisión de implementar un sistema CRM, deriva la necesidad de establecer una alianza con al menos un Partner tecnológico de referencia y suficiente experiencia y arraigo en el sector de la Construcción española. A través de esta alianza se pretenden conseguir varios aceleradores del proyecto:

- Seleccionar la muestra apropiada de empresas. 
- Favorecer la ratio de respuesta al formulario.

A continuación, se definen las líneas maestras de este plan de trabajo.

\subsection{ELECCIÓN DE LOS DATOS Y LA MUESTRA MEDIANTE ALIANZA CON UN PARTNER TECNOLÓGICO ESPECIALIZADO}

El paso previo a la elección del método de análisis de resultados es conocer la población disponible para realizar el estudio empírico, así como los detalles de la muestra con la que se va a trabajar. Con el fin de validar empíricamente el impacto del uso de CRM en el desempeño de la empresa en el sector de la Construcción español, se ha de identificar el segmento más representativo dentro de dicho sector de la Construcción en España.

Este proyecto pretende aportar no solo una evidencia empírica y científica del cumplimiento de las expectativas sobre los beneficios esperados del uso de CRM (Gil-Gomez et al., 2020), sino que pretende también (e incluso principalmente) obtener argumentos de valor y de referencia para que los Partners tecnológicos que trabajan en el ámbito de la transformación digital de las empresas de este sector. Es por ello que ya de inicio en este proyecto se involucra a este tipo de Partners, con el objetivo de:

- Identificar el ámbito de estudio que pueda tener mayor impacto en las empresas del sector.

- Establecer una alianza de colaboración que permita una mejor llegada a las empresas que formen parte de la muestra de estudio.

Tras entrevistar a algunos de los Partners tecnológicos con mayor número de clientes en el sector de la construcción en España, y atendiendo a su conocimiento y experiencia en el sector, así como a los estudios más recientes al respecto (Marquesme, 2021) se ha seleccionado una muestra listada de empresas que incluye aquellas que estos Partners tecnológicos proponen como:

- Las que mayor impacto deberían observar en sus indicadores de desempeño organizacional en caso de usar CRM. 
- Las que disponen de estructuras organizativas y de propiedad más favorables a los proyectos de adopción de soluciones de CRM.

Atendiendo pues a esta propuesta, y para poder realizar una validación empírica del impacto de uso de CRM, el estudio ha seleccionado como población objeto de estudio empresas nacionales del sector de la Construcción e Inmobiliario con una facturación anual en el rango de 10 a 200 millones de euros, y registradas en el Instituto Nacional de Estadística (INE) con el siguiente GNAE:

\section{PROMOCIÓN INMOBILIARIA}

4121. GONSTRUGGIÓN DE EDIFICIOS RESIDENGIALES

4122. GONSTRUGCIÓN DE EDIFICIOS NO RESIDENGIALES

4211. GONSTRUCGIÓN DE GARRETERAS Y GARRETERAS

4212. CONSTRUCGIÓN DE VÍAS SUPERFICIALES Y SUBTERRÁNEAS

4213. CONSTRUGGIÓN DE PUENTES Y TÚNELES

4221. CONSTRUCGIÓN DE REDES PARA FLUIDOS

4222. CONSTRUGCIÓN DE REDES ELÉGTRIGAS Y DE TELEGOMUNICACIONES 4299. CONSTRUCGIÓN DE OTROS PROYECTOS DE INGENIERÍA GIVIL

Mediante el estudio del impacto de uso de CRM sobre el desempeño organizacional en esta muestra, se tratará de aportar argumentos de impacto que ayuden a los tomadores de decisión de las empresas que aún no han decidido aportar por CRM como solución tecnológica clave para su gestión de relaciones con sus clientes. 


\subsection{ELECCIÓN DE LAS VARIABLES}

Atendiendo a estudios previos sobre diferentes modelos de validación empírica sobre el impacto de uso de CRM (Guerola-Navarro et al., 2020b; Valmohammadi, 2017; Calantone et al., 2002; Zegarra, 2014), se ha considerado elegir las siguientes variables de studio:

- Grado de uso de GRM.

- Capacidad de Innovación.

- Mejoras en el Desempeño Organizacional.

Analizados los diferentes modelos, en los referenciados entre los estudios más relevantes publicados en revistas de prestigio, se eligen estas variables como las que mayor se adecúan al objeto último que persigue el presente estudio.

En primer lugar, por Grado de uso de CRM entendemos (Valmohammadi, 2017) en qué medida utiliza la potencia de gestión de CRM aquella empresa que tiene y usa un sistema CRM de cualquier tipo y fabricante. En concreto, Valmohammadi (2107) propone medir el grado de uso de CRM a través de los siguientes elementos identificativos del uso de características y herramientas de CRM:

- El intercambio de información.

- Involucramiento del cliente.

- Asociación a largo plazo.

- Resolución conjunta de problemas.

- CRM basado en tecnología.

En referencia a la segunda variable, la Capacidad de Innovación, también identificada en general como "Innovación", Calantone et al. (2002) la define como la capacidad que tiene la empresa de adaptarse a nuevas circunstancias de Mercado, tanto si vienen de parte del producto (bienes y servicios ofertados al mercado) como si vienen de part de los procesos internos (tanto procesos productivos como de gestion. 
Calantone et al. (2002) propone una escala de medición de la capacidad de innovación a través de los siguientes elementos:

- Nuestra empresa prueba con frecuencia nuevas ideas.

- Nuestra empresa busca nuevas formas de hacer las cosas.

- Nuestra empresa es creativa en sus métodos de operación.

- Nuestra empresa es a menudo la primera en comercializar nuevos productos y servicios.

- La innovación en nuestra empresa se percibe como demasiado arriesgada y se resiste.

- Nuestra introducción de nuevos productos ha aumentado en los últimos 5 años.

Finalmente, como variable de impacto que se propone para medir cómo se puede esperar que el uso de CRM mejore los resultados empresariales, Zegarra (2014) propone el Desempeño Organizacional. Este modelo utiliza la escala de Nakata et al. (2008), que compara el desempeño de la organización con el de otros competidores y se compone de los siguientes ítems:

- La calidad del producto o servicio.

- $\quad$ El éxito de nuevos productos o servicios.

- La tasa de retención de clientes.

- El nivel de ventas.

- La rentabilidad del capital.

- Margen de utilidad bruta.

- El retorno de la inversión

Una vez identificadas las variables de medición que puedan relacionar el impacto de uso de CRM sobre el desempeño organizacional, con la intervención de la capacidad de innovación, el siguiente paso será construir un formulario de recogida de datos entre las empresas de la muestra, y seleccionar un modelo de análisis de las respuestas obtenidas. 


\subsection{CUESTIONARIO Y METODOLOGÍA DE ANÁLISIS DE DATOS}

Con el objeto de recoger los datos que den lugar a conclusiones sobre el impacto del uso de CRM en las empresas del sector de la construcción en España, se propone el diseño de un formulario en función de constructos e ítems que ya hayan sido validados previamente en la literatura científica. De entre las alternativas revisadas en la literatura, se ha elegido tomar como base el cuestionario de Guerola-Navarro et al. (2020b), cuyo carácter general y objetivo hace que sea idóneo para el actual proyecto con la adición de dos nuevas secciones específicas para el sector en estudio.

El cuestionario propuesto cuenta con las 7 secciones que se muestran en la Figura 3, de las cuales las dos últimas son fruto de la aportación del Partner tecnológico por su conocimiento del sector.

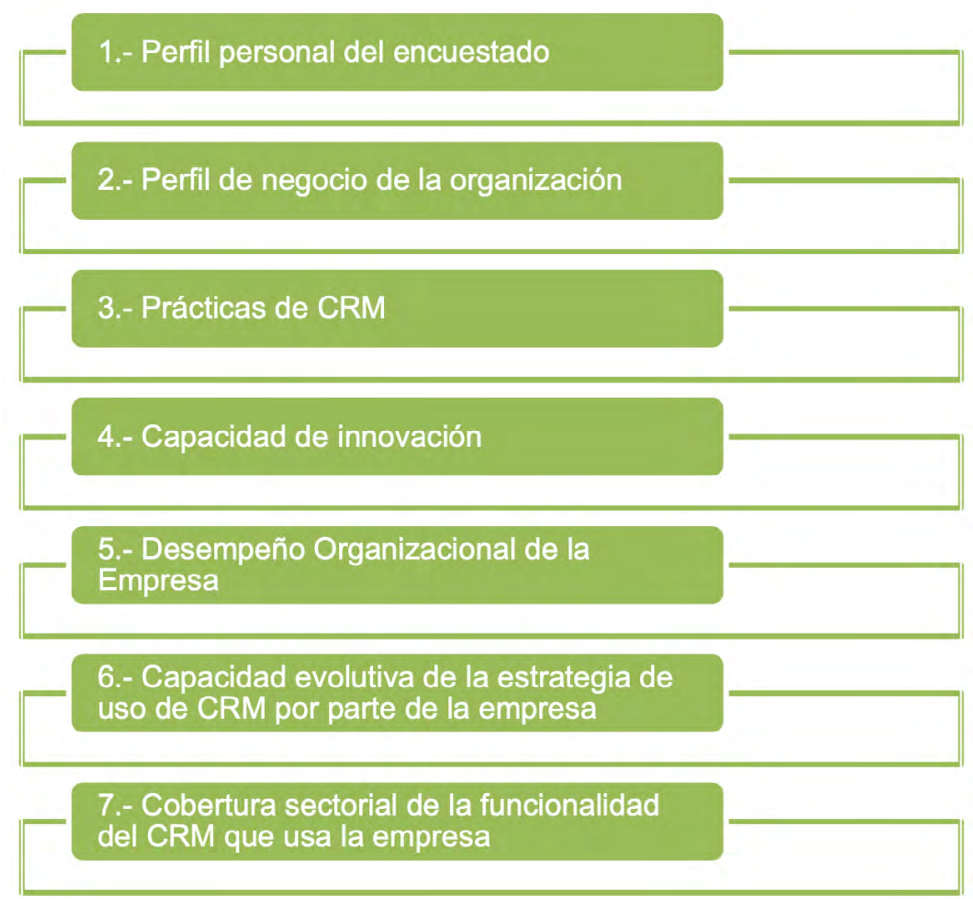

Figura 3. Secciones del formulario de recogida de datos.

Fuente: elaboración propia. 
En cuanto a la metodología a seguir para analizar las respuestas obtenidas a través del cuestionario, se propone elegirla en función del volumen de respuestas obtenidas a través del formulario. Los diferentes enfoques cuantitativos, cualitativos, y mixtos (Sampieri, 2018) dan multitud de alternativas para que la metodología de análisis de los datos sea acorde a la representatividad de la muestra poblacional elegida y la ratio de respuesta al cuestionario.

\section{RESULTADOS Y ESTADO ACTUAL DEL PROYECTO}

Como resultado de las acciones llevadas a cabo, y siguiendo el orden de cada una de las etapas del Plan de Trabajo propuesto, se ha llegado a los siguientes resultados:

1. Se ha elegido un Partner tecnológico con elevado prestigio y gran penetración de mercado en el área de las Tecnologías de Información y Comunicación (TIC), que tiene el mayor número de referencias de clientes del sector de la construcción en España, y que cuenta con un equipo y una estrategia específica y diferenciada para dicho sector. Se ha establecido una alianza con este Partner para colaborar de forma desinteresada en la ejecución del proyecto.

2. Se ha elegido la muestra de empresas que van a participar en el estudio del grado de impacto del uso de CRM sobre el desempeño organizacional. Esta selección de empresas se ha realizado en colaboración con el Partner estratégico, atendiendo a criterios de CNAE (código estadístico de actividad económica registrado oficialmente en el Instituto Nacional de Estadística) y a criterios de facturación.

3. Se han seleccionado las variables que se consideran relevantes en el estudio, siguiendo los estudios científicos previos publicados. En concreto se han elegido 3 variables: Grado de uso de CRM, Capacidad de Innovación, y Desempeño Organizacional.

4. Se ha construido un formulario para la recogida de datos, en base a un cuestionario previamente testeado y validado científicamente y empíricamente. Sobre este cuestionario se ha añadido 
dos secciones recomendadas por el Partner tecnológico de referencia. En el momento actual el proyecto se encuentra en fase de recogida de respuestas a través de un formulario web.

5. Queda por definir, en función de la ratio de respuesta, cual será la metodología más apropiada para analizar los datos obtenidos.

6. Se espera obtener unos resultados y conclusiones que aporten criterios de decisión valiosos a los Tomadores de Decisión de las empresas del sector de la Construcción que deban valorar la idoneidad de afrontar proyectos de implementación de soluciones CRM.

\section{CONCLUSIONES}

El presente artículo muestra el objetivo, plan de trabajo, y estado actual del proyecto CRM-CONSTRES, cuyo objetivo fundamental es analizar el grado de adopción de las soluciones tecnológicas CRM por parte de las empresas del sector de la Construcción en España, así como obtener conclusiones sobre el impacto que sobre el desempeño organizacional puede tener el uso de dichas soluciones CRM.

Por la especificidad del sector en estudio, así como por sus especiales características, se ha buscado una alianza estratégica con un Partner tecnológico altamente especializado en el sector. Siguiendo las directrices del método científico, y con la aportación de la experiencia y conocimiento del Partner estratégico, se ha avanzado en la línea de la definición de objetivos, muestra y listado de empresas en estudio, selección de las variables de análisis, y construcción de un formulario para la recogida de datos.

Como futura línea de investigación, y en una fase posterior a la conclusión de la recogida de respuestas al formulario, el análisis de dichas respuestas a través de la metodología más apropiada a la muestra elegida deberá de proporcionar elementos de juicio y decisión a las empresas del sector construcción que en un futuro se planteen desplegar alguna solución CRM. 


\section{REFERENCIAS BIBLIOGRÁFICAS}

AlQershi, N.A., Mokhtar, S.S.M., y Abas, Z.B. (2020). GRM dimensions and performance of SMEs in Yemen: the moderating role of human capital. Fournal of Intellectual Capital, Vol. ahead-of-print No. ahead-of-print. https://doi.org/10.1108/JIC-05-2020-0175

Araújo, G. G. S. D., Pedron, G. D., y Picoto, W. N. (2018). What's behind CRM research? A bibliometric analysis of publications in the CRM research field. Fournal of Relationship Marketing, 17(1), 29-51. https://doi.org/10.1080/15332667.2018.1440139

Belias, D., Velissariou, E., Kyriakou, D., Vasiliadis, L., Mantas, C., Sdrolias, L., ... y Kakkos, N. (2018). The importance of customer relationship management and social media in the Greek wine tourism industry. In Innovative approaches to tourism and leisure (pp. 249-259). Springer, Cham.

Buttle, F. (2004). Customer Relationship Management. Concepts and Tools. Elsevier Butterworth-Heinemann.

Calantone, R. J., Gavusgil, S. T., y Zhao, Y. (2002). Learning orientation, firm innovation capability, and firm performance. Industrial marketing management, 31(6), 515-524. https://doi.org/10.1016/ S0019-8501(01)00203-6

Chen, I.J., y Popovich, K. (2003). Understanding customer relationship management (GRM) People, process and technology. Business Process Management Fournal, 9(5), 672-688. https://doi. org/10.1108/14637150310496758

Grescimanno, M., Galati, A., Tulone, A., y Tinervia, S. (2017). Social media technology use and managers perception. A preliminary study in the italian wine industry. En 10th Annual Conference of the EuroMed Academy of Business. 
Dew, N., Read, S., Sarasvathy, S. D., y Wiltbank, R. (2011). On the entrepreneurial genesis of new markets: effectual transformations versus causal search and selection. Fournal of Evolutionary Economics, 21(2), 231-253. https://doi.org/10.1007/s00191-010-0185-1

Ferrer-Lorenzo, J. R., Abella-Garcés, S., y Maza-Rubio, T. (2017). Competitive advantage differences between firms belonging to a business group and independent companies in the Spanish wine industry. Economía Agraria y Recursos Naturales-Agricultural and Resource Economics, 17(2), 105-132. https://doi.org/10.7201/earn.2017.02.05

Gil-Gomez, H., Guerola-Navarro, V., Oltra-Badenes, R., y Lozano-Quilis, J. A. (2020). Customer relationship management: digital transformation and sustainable business model innovation. Economic Research-Ekonomska Istraživanja, 33(1), 2733-2750. https://doi.org/10.1080/ 1331677X.2019.1676283

Guerola-Navarro, V., Gil-Gomez, H., Oltra-Badenes, R., y Sendra-García,J. (2021a). Customer relationship management and its impact on Innovation: A literature review. Fournal Of Business Research, 129, 83-87. https://doi.org/10.1016/j.jbusres.2021.02.050

Guerola-Navarro, V., Oltra-Badenes, R., Gil-Gomez, H., y Gil-Gomez, J. A. (2020a). Customer relationship management (CRM): a bibliometric analysis. International Fournal of Services Operations and Informatics, 10(3), 242-268. https://doi.org/10.1504/IJSOI.2020.108988

Guerola-Navarro, V., Oltra-Badenes, R., Gil-Gomez, H., y Gil-Gomez, J. A. (2020b). Research model for measuring the impact of Customer Relationship Management (CRM) on performance indicators. Economic Research-Ekonomska Istraživanja, 34(1). https://doi.org/10.1080/133167 7X.2020.1836992 
Guerola-Navarro, V., Oltra-Badenes, R., Gil-Gomez, H., y Iturricha Fernández, A. (2021b). Customer relationship management (CRM) and Innovation: A qualitative comparative analysis (QCA) in the search for improvements on the firm performance in winery sector. Technological Forecasting \& Social Change, 169, 120838. https://doi.org/10.1016/j.techfore.2021.120838

Guerola-Navarro, V., Oltra-Badenes, R., y Gil-Gómez, H. (2020c). Análisis de la relación entre el grado de introducción de CRM y los beneficios de la empresa a través del desempeño organizacional y la innovación empresarial. 3C Empresa: investigación y pensamiento crítico, 9(1), 67-87. http://doi.org/10.17993/3cemp.2020.090141.67-87

Gutierrez, P. K., y Romero, D. R. (2021). Evolución del modelo de mejora continua en la atención al cliente: una revisión de la literatura científica los últimos 10 años.

Huang, D.Y., y Lin, G.Y. (2005). Customer-oriented financial service personalization. Industrial Management and Data systems, 105(1), 26-44. https://www.emerald.com/insight/content/ doi/10.1108/02635570510575171/full/html

Iazzi, A., Trio, O., Fait, M., y Iaia, L. (2013). Social Web Communication and CRM in the marketing strategies of wine enterprises. International Journal of Economic Behavior, 3(1), 103-116. https://ideas. repec.org/a/but/ijebfa/v3y2013i1p103-116.html

Imhoff, G., Loftis, L., y Geiger, J. G. (2001). Building the customer-centric enterprise: Data warehousing techniques for supporting customer relationship management. Wiley.

Izquierdo, G. G., Gillán, J. G., y Gutiérrez, S. S. M. (2005). The impact of customer relationship marketing on the firm performance: a Spanish case. Journal of Services Marketing, 19(4), 234-244. https://www.emerald.com/insight/content/doi/10.1108/08876040510605262/full/html 
Jocovic, M., Melovic, B., Vatin, N., y Murgul, V. (2014). Modern business strategy Customer Relationship Management in the area of civil engineering. En Applied Mechanics and Materials (Vol. 678, pp. 644-647). Trans Tech Publications Ltd.

Joo, J. (2007). An empirical study on the relationship between customer value and repurchase intention in Korean internet shopping malls. The fournal of Computer Information Systems, 48(1), 53-62. https:// www.tandfonline.com/doi/abs/10.1080/08874417.2007.11645995

Kane, G. G., Palmer, D., Phillips, A. N., Kiron, D., y Buckley, N. (2015). Strategy, not technology, drives digital transformation. MIT Sloan Management Review and Deloitte University Press, 14(1-25). https://www2.deloitte.com/cn/en/pages/technology-media-and-telecommunications/articles/ strategy-not-technology-drives-digital-transformation.html

Kurniawan, R., Juliana, A. A., Brizal, H., Vianthoni, G. R., y Hadiyanti, F. J. (2021). Relations with the Implementation of Relationship Marketing on Customer Loyalty: A Study on Café Resto Blackpepper Bandung. Psychology and Education Fournal, 58(1), 6033-6037.

Marquesme. (2021). Digitalización del sector de la construcción en España. https:// marquesme.com/recursos/ digitalizacion-sector-construccion-espana

Melovic, B., Jocovic, M., Lugovskaya, I., y Vatin, N. (2015). Possibilities of implementing customer relationship management in the function of improving the competitiveness of the civil engineering sector. En Applied Mechanics and Materials (Vol. 725, pp. 977-983). Trans Tech Publications Ltd.

Nakata, C., Zhu, Z., y Kraimer, M. L. (2008). The complex contribution of information technology capability to business performance. Fournal of Managerial Issues, XX(4), 485-506. https: / / www.jstor. org/stable/40604625

Nambisan, S. L., Majchrzak, K., y Song, A. M. (2017). Digital innovation management: Reinventing innovation management research in a digital world. MIS Quarterly, (1), 41. 
Okrepilov, V. V., y Melovic, B. (2015). Modern business models of the competitiveness enhance of the construction sector enterprises. Stroitel'stvo Unikal'nyh Zdanij i Sooruzenij, (10), 95.

Preece, C., Ghong, H. Y., Golizadeh, H., y Rogers, J. (2015). A review of customer relationship (CRM) implications: benefits and challenges in construction organizations. International fournal of Civil Engineering, 13(3), 362-371. https://www.researchgate.net/publication/283107497_A_ review_of_customer_relationship_CRM_implications_Benefits_and_challenges_in_ construction_organizations

Rahmadi, A. N., Djunaedi, D., y Nurlaely, N. (2021). The Effect of Customer Relationship Management (CRM) and Entrepreneurship Orientation Towards the Company Performance in Micro Small Medium Enterprises in Kediri. En 2nd International Conference on Business and Management of Technology (ICONBMT 2020) (pp. 65-68). Atlantis Press. https://doi.org/10.2991/ aebmr.k.210510.013

Ramanathan, U., Subramanian, N., Yu, W., y Vijaygopal, R. (2017). Impact of customer loyalty and service operations on customer behaviour and firm performance: empirical evidence from UK retail sector. Production Planning $\&$ Control, 28(6-8), 478-488. https://www.researchgate.net/ publication/324483470_Impact_of_customer_loyalty_and_service_operations_on_customer_ behaviour_and_firm_performance_empirical_evidence_from_UK_retail_sector

Ribeiro-Navarrete, S., Saura, J. R., y Palacios-Marqués, D. (2021). Towards a new era of mass data collection: Assessing pandemic surveillance technologies to preserve user privacy. Technological Forecasting and Social Change, 167, 120681. https://doi.org/10.1016/j.techfore.2021.120681

Sampieri, R. H. (2018). Metodología de la investigación: las rutas cuantitativa, cualitativa y mixta. McGraw Hill México. 
Sear, E. A., Hartland, T. G., Abdel-Wahab, M. S., y Miller, G. G. (2007). Customer Relationship Management Implementation: A Case Study in the Construction Sector. https://citeseerx.ist.psu.edu/ viewdoc/download?doi=10.1.1.559.4599\&rep=rep1\&type=pdf

Valmohammadi, G. (2017). Customer relationship management: Innovation and performance. International Fournal of Innovation Science, 9(4), 374-395. https:// doi.org/10.1108/IJIS-02-2017-0011

Vicedo, P., Gil-Gomez, H., Oltra-Badenes, R., y Guerola-Navarro, V. (2020) A bibliometric overview of how critical success factors influence on enterprise resource planning implementations. Journal of Intelligent \& Fuzzy Systems, 38(5), 5475-5487. https://doi.org/10.3233/JIFS-1 79639

Wahlberg, O., Strandberg, G., Sundberg, H., y Sandberg, K. W. (2009). Trends, topics and underresearched areas in CRM research: a literature review. International fournal of Public information systems, 3, 191-208. https://www.diva-portal.org/smash/get/diva2:272876/FULLTEXT01.pdf

Zegarra, A. (2014). La orientación al mercado y el efecto de la adopción de tecnologías Web 2.0 y el aprendizaje organizativo en la capacidad de innovación: estudio empírico en empresas del sector hotelero de España (Doctoral dissertation). 
